\title{
COLLAPSE PRESSURE ESTIMATES AND THE APPLICATION OF A PARTIAL SAFETY FACTOR TO CYLINDERS SUBJECTED TO EXTERNAL PRESSURE
}

\author{
YEON-SIK YOO ${ }^{1}$, NAM-SU HUH ${ }^{2 *}$, SUHN CHOI' ${ }^{1}$ TAE-WAN KIM ${ }^{1}$ and JONG-IN KIM ${ }^{1}$ \\ ${ }^{1}$ Korea Atomic Energy Research Institute \\ 1045 Daedeok Street, Yuseong-gu, Daejeon 305-353, Republic of Korea \\ ${ }^{2}$ School of Mechanical Design and Automation Engineering \\ Seoul National University of Technology \\ 172 Gongreung 2-dong, Nowon-gu, Seoul 139-743, Republic of Korea \\ *Corresponding author. E-mail : nam-su.huh@snut.ac.kr
}

Received February 26, 2010

Accepted for Publication May 31, 2010

The present paper investigates the collapse pressure of cylinders with intermediate thickness subjected to external pressure based on detailed elastic-plastic finite element (FE) analyses. The effect of the initial ovality of the tube on the collapse pressure was explicitly considered in the FE analyses. Based on the present FE results, the analytical yield locus, considering the interaction between the plastic collapse and local instability due to initial ovality, was also proposed. The collapse pressure values based on the proposed yield locus agree well with the present FE results; thus, the validity of the proposed yield locus for the thickness range of interest was verified. Moreover, the partial safety factor concept based on the structural reliability theory was also applied to the proposed collapse pressure estimation model, and, thus, the priority of importance of respective parameter constituting for the collapse of cylinders under external pressure was estimated in this study. From the application of the partial safety factor concept, the yield strength was concluded to be the most sensitive, and the initial ovality of tube was not so effective in the proposed collapse pressure estimation model. The present deterministic and probabilistic results are expected to be utilized in the design and maintenance of cylinders subjected to external pressure with initial ovality, such as the once-through type steam generator.

KEYWORDS : Elastic Instability, External Pressure, Ovality, Partial Safety Factor, Load and Resistance Factor Design, Advanced First-Order Second Moment, Target Probability of Failure, Most Probably Point, Plastic Collapse, Yield Locus

\section{INTRODUCTION}

Recently, cylinders subjected to external pressure have become a point of interest in the nuclear industry due to their application to advanced small and medium-sized light water reactors. For instance, once-through type steam generator (SG) tubes, in which primary coolant flows outside the tubes while secondary water flows inside the tubes, consist of typical examples of cylinders under external pressure. Another example would be pipelines under seawater to transport gas, oil, etc. In order to provide guidance on a design and integrity evaluation of a cylinder under external pressure, the failure characteristics of a cylinder under external pressure should carefully be considered. The failure mechanisms of such a cylinder might be quite different from those of a typical one under internal pressure. It is well known that the failure mechanism of a cylinder under external pressure depends on the geometries of the cylinder, i.e. the ratio of diameter to thickness [1]. In the case of a thin-walled cylinder, the failure of the cylinder is dominated by elastic buckling (elastic instability), for which the assessment methods have been well established and many numerical and experimental works have been conducted [2,3]. When the thickness of a cylinder subjected to external pressure becomes larger, the failure mechanism of such a cylinder is changed to plastic collapse rather than elastic buckling. Upon the estimation of load carrying capacity of this thick-walled cylinder under external pressure and combined loading, many numerical and experimental works have been also made to propose relevant design criteria of thick-walled cylinders subjected to external pressure [4 6].

On the other hand, recently, a helically coiled cylinder with an intermediate thickness range has been applied to the steam generator tubes of newly developed nuclear plants, i.e. advanced integral reactor [7]; thus, accurate guidance 
to investigate collapse behaviour would be needed for a cylinder with intermediate thickness. The failure of cylinders under external pressure with intermediate thickness might be influenced by plastic yielding. At this point, another notable point is that the imperfection, such as tube ovality, would inherently be produced during the tube coiling process to fabricate a steam generator consisting of helically coiled tubes. In such cases it is probably realistic to treat not only the plastic collapse but also inelastic local buckling due to ovality as an important failure mechanism; that is, the interaction of plastic collapse and local instability should be considered. In order to explicitly consider such an interaction effect due to local imperfection, a detailed non-linear finite element (FE) analysis might be required.

Another important issue relating to the design and FFS (Fitness-For-Service) assessment of nuclear components is the application of reliability or risk based concepts to evaluate the parametric sensitivity and to improve safety and reduce of cost. For the newly developed nuclear reactors, it is crucial to establish a reliability-based design methodology to take the statistical importance of each design variable into account. Although, deterministic failure assessment has been successfully applied in demonstrating the stability of nuclear components, key ingredients in failure assessment, geometric variables (diameter, thickness, ovality parameter, etc.) and the mechanical properties of material of interest affecting assessment results, are often exposed to considerable uncertainty; thus, a statistical or probabilistic approach to the structural integrity of the nuclear components has been introduced (for example, see Ref. [8, 9]), which is also of great interest in risk-based regulatory, design, and maintenance. In particular, the application of a reliability assessment incorporating a partial safety factor (PSF) concept, in which the influence of each variable can be evaluated separately for a certain target probability, makes it possible to realize an optimized design with proper margins under the reliability level [10 12]. Recently, ASME Sec. III code has examined the application of the PSF, which gives an equivalent failure probability compared to the present design method, to nuclear piping design. The PSF concept can also offer the information to determine the priority of importance of each variable, and, thus, the respective PSF can control each other for the target probability in the view of design and FFS assessment.

This paper presents a collapse pressure estimation model for cylinders subjected to external pressure considering the interaction of plastic collapse and local instability due to the ovality. Based on the proposed estimation model and reliability assessment using the PSF concept, the priority of importance of each design variable was investigated. For this purpose, the PSFs of each variable to constitute the collapse pressure estimation model were evaluated using the AFOSM (Advanced First-Order Second Moment) method for the postulated target probability. Section 2 describes the FE analyses performed in the present study.
The proposed yield locus to evaluate the collapse pressure of the cylinder under external pressure considering the interaction of plastic collapse and local instability due to ovality and the validation results are given in Section 3 , and the reliability evaluation based on the PSF concept are given in Section 4. The present work is discussed in Section 5 and concluded in Section 6.

\section{FINITE ELEMENT ANALYSES}

\subsection{Geometry}

In the present study, two geometries, i.e. a perfect cylinder and cylinder with initial ovality, were considered to investigate the effect of ovality on the collapse pressure and to propose a yield locus considering the interaction of plastic collapse and local instability due to ovality. Figure 1 depicts the geometry of a cylinder subjected to external pressure where $D_{\mathrm{o}}, D_{\mathrm{i}}, D_{\mathrm{m}}$, and $t$ are the outer diameter, inner diameter, mean diameter, and cylinder thickness, respectively. In the present study, the outer diameter and thickness of the cylinder considered were $17 \mathrm{~mm}$ and $2.5 \mathrm{~mm}$, which are identical to those of a South Korean integral reactor under development; thus, the diameter and thickness ratio was 6.8 , which corresponds to an intermediate thickness range. In addition, to quantify the effect of thickness on the collapse pressure due to the interaction of plastic collapse and local instability and to investigate the possibility to reduce the thickness of the cylinder, another case with intermediate thickness, i.e. the case of $D_{\mathrm{o}} / t=10$, was also considered.
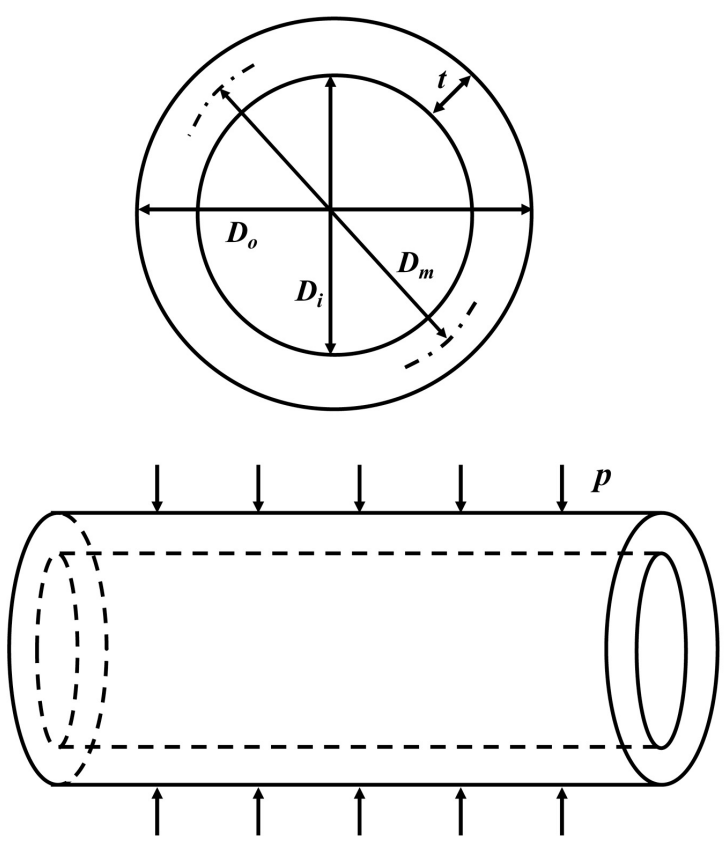

Fig. 1. Schematics of Cylinder Under External Pressure 
As mentioned above, in order to propose a yield locus considering the interaction of plastic collapse and local instability due to ovality, four different values of ovality were considered. In the present study, the ovality of the cylinder was defined as follows:

$$
W=\frac{D_{o, \max }-D_{o, \min }}{D_{o}}
$$

where $D_{\text {o,max }}$ and $D_{\text {o,min }}$ denote the maximum and minimum values of the outer diameter of cylinder with ovality, respectively, and $D_{\mathrm{o}}$ is the outer diameter of a perfect cylinder. The definition of each ovality variable is shown in Fig. 2, and analyses cases are summarized in Table 1, where the case of $W=0$ corresponds to a perfect cylinder, i.e. cylinder without ovality.

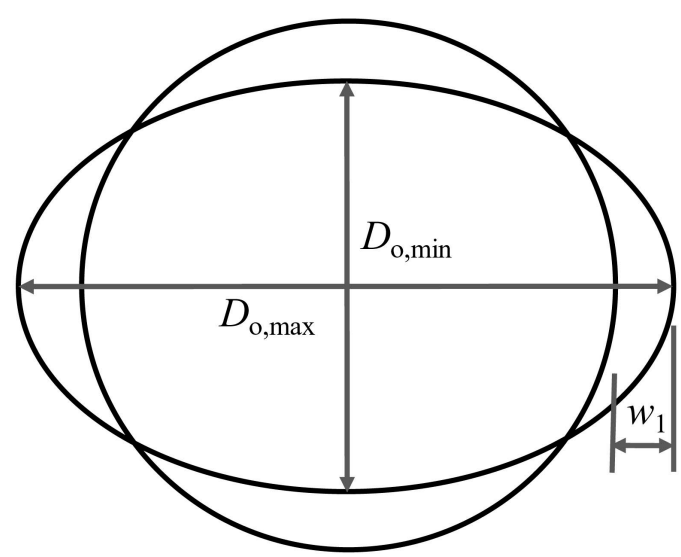

Fig. 2. Cross Section of Cylinder with Ovality

The displacement due to ovality is defined as follows:

$$
w_{1}=\frac{W D_{o}}{4} \text {. }
$$

Thus, a total of eight cases, four different values of initial ovality and two different values of the ratio of outer diameter to thickness $\left(D_{\mathrm{o}} / t\right)$, were considered to evaluate the effect of bending moment due to initial ovality on the collapse pressure and to validate the proposed yield locus considering the interaction of instability and plastic collapse in the present study.

\subsection{Finite Element Analysis}

In order to take into account the effect of local instability due to imperfection on the collapse pressure, a detailed 3-dimensional (3-D) non-linear FE analyses should be carried out. In this context, elastic-perfectly plastic analyses of the FE models for the perfect cylinder and cylinder with initial ovality, depicted in Figs. 1 and 2, were performed using the general-purpose FE program, ABAQUS [13]. Material was assumed to be elastic-perfectly plastic with a Poisson's ratio ( $v$ ) of 0.29 . The steam generator tubes were made of Alloy 690, and they are operated at the operating temperature of $360^{\circ} \mathrm{C}$. The yield strength $\left(\sigma_{y}\right)$ and Young's modulus $(E)$ of Alloy 690 at this operating temperature were $212 M P a$ and $191 G P a$, respectively, which can be obtained from Ref. [14]. The non-hardening $J_{2}$ flow theory was employed using a large geometry change continuum FE model to consider local instability due to ovality.

Due to symmetric conditions, only half of the cylinder was modeled to reduce computing time. In the present cylinder model with initial ovality, the deformation and relevant collapse are dominated not by general elastic buckling but by local plastic collapse due to local initial ovality (symmetric ovality). Therefore, the symmetric FE models were employed, which means that non-symmetric deformation might not be produced. Figure 3 shows a typical 3-D FE mesh employed in the present work. For the FE model, reduced integration isoparametric 20-node brick elements (element type C3D20R in ABAQUS element library) were used. Moreover, to avoid problems associated with convergence in elastic-perfectly plastic calculations, the modified RIKS option within ABAQUS was invoked. The numbers of elements and nodes in the FE mesh are 78200 elements/366293 nodes. To accurately capture the

Table 1. Summary of Geometric Variables and Material Properties Considered in the Present Study

\begin{tabular}{c|c|c|c|c}
\hline $\begin{array}{c}D_{\mathrm{o}} \\
(\mathrm{mm})\end{array}$ & $D_{\mathrm{o}} / t$ & $\begin{array}{c}W \\
\left(=\frac{D_{o, \max }-D_{o, \min }}{D_{o}}\right)\end{array}$ & $\begin{array}{c}\sigma_{y} \\
(M P a)\end{array}$ & $\begin{array}{c}E \\
(G P a)\end{array}$ \\
\hline \multirow{2}{*}{17} & $6.8,10$ & $\begin{array}{c}0,0.025, \\
0.03,0.05\end{array}$ & 212 & 191 \\
\hline
\end{tabular}


stress distribution through the thickness, five elements were used through the wall thickness of the cylinder. External pressure was applied as a distributed load to the outer surface of the FE model. Since the actual steam generator tubes are long enough and fixed by the tube supports, both ends of the cylinder were fully constrained. The length of the FE model is ten times the outer diameter of a perfect cylinder.

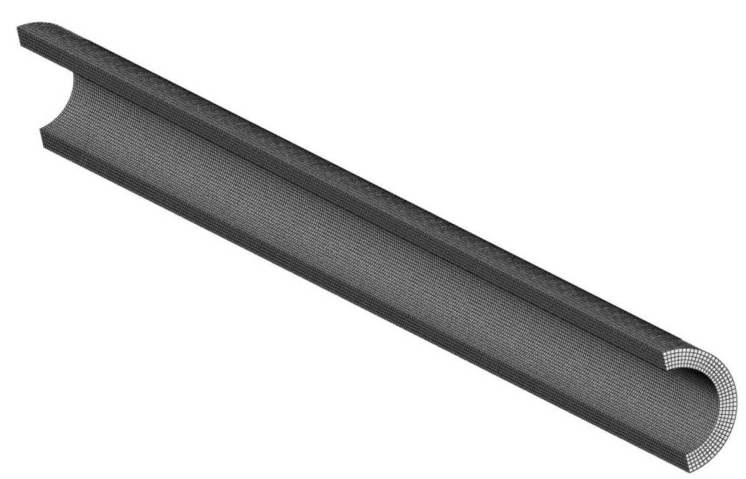

Fig. 3. Typical FE Mesh Employed in the Present Work

\section{PROPOSED YIELD LOCUS AND RESULTS}

\subsection{Yield Locus for a Cylinder with Initial Ovality under External Pressure}

As noted previously, the initial ovality made during the tube coiling process of a cylinder under external pressure leads to bending moment. Thus, in order to estimate the collapse pressure accurately and propose a relevant yield locus for such a cylinder, this combined loading should be considered. In the present study, to propose the yield locus considering the interaction between plastic collapse and local instability and combined external pressure and bending moment due to initial ovality based on the von Mises yield condition, the following admissible form for the yield locus was postulated:

$$
\left(\frac{p}{p_{L}^{\text {Mises }}}\right)^{2}+\left(\frac{M}{M_{L}^{\text {Mises }}}\right)=1
$$

where $p$ is the applied external pressure. $p_{\mathrm{L}}$ denotes the plastic collapse pressure of a perfect cylinder, and $M_{\mathrm{L}}$ denotes the plastic collapse moment of a beam, and the superscription 'Mises' means the plastic collapse load based on the von Mises yield condition. Thus, it can be postulated that the yield locus of Eq. (3) provides an accurate Mises yield locus under combined external pressure and bending moment due to initial ovality.
In the present study, it is assumed that the following expression of the plastic collapse pressure of a perfect cylinder, $p_{L}^{\text {Mises }}$, can be used [15]. Note that the factor $2 / \sqrt{3}$ is due to the von Mises condition.

$$
p_{L}^{\text {Mises }}=\frac{2}{\sqrt{3}} \sigma_{y} \ln \left(\frac{D_{o}}{D_{i}}\right)
$$

Although, the plastic collapse pressure of Eq. (4) is derived for a thick-walled cylinder under internal pressure, this solution also can be applied to a thick-walled cylinder under external pressure to obtain fully plastic collapse pressure. The present FE collapse pressure $\left(p_{c}\right)$ of a perfect cylinder under external pressure is compared with the results from Eq. (4) in Fig. 4. As shown in Fig. 4, the present results agree very well with the solution from Eq. (4); thus, the confidence of the present FE model and application of Eq. (4) to the cylinder under the external pressure is gained.

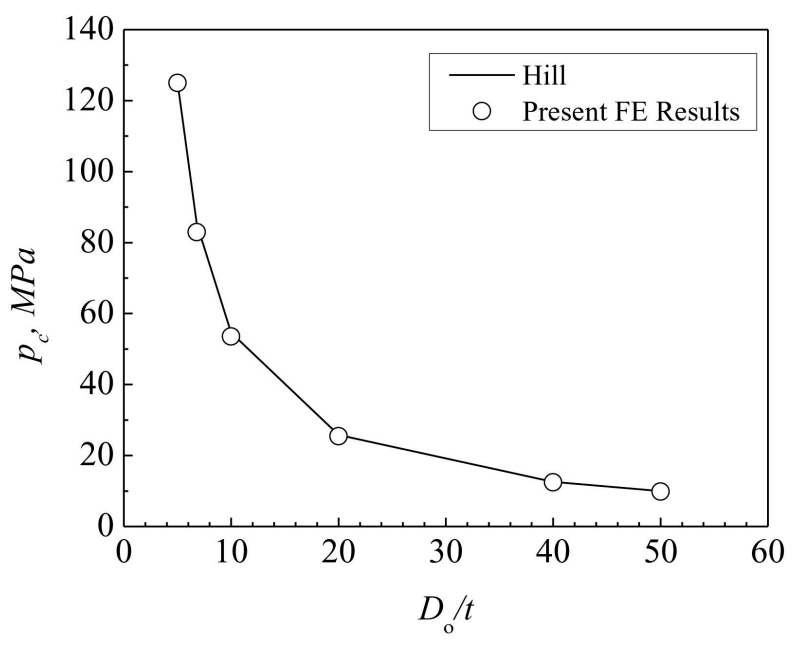

Fig. 4. Comparison of the FE Collapse Pressure for a Perfect Cylinder with Hill's Solution

In Eq. (3), as a plastic collapse moment, $M_{L}^{\text {Mises }}$, the following expression for the plastic collapse moment of a beam can be utilized.

$$
M_{L}^{\text {Mises }}=\frac{\sigma_{y} t^{2}}{2 \sqrt{3}}
$$

More importantly, the expression for the bending moment, $M$, due to initial ovality and external pressure can be easily obtained from Ref. [16]: 


$$
\begin{gathered}
M=M_{I} \frac{1}{1-\frac{p}{p_{E}}} \\
M_{I}=\frac{1}{2} p D_{o} w_{1}=\frac{1}{8} p D_{o}^{2} W \\
\left.p_{E}=2 \frac{E}{1-v^{2}} \frac{D_{o}}{\frac{D_{o}}{t}}-1\right)^{2}
\end{gathered}
$$

where $w_{1}\left(=\frac{W D_{o}}{4}\right)$ is the displacement due to initial ovality (see Fig. 2).

\subsection{Results}

Figure 5 shows the deformed shape of a cylinder with initial ovality under external pressure for the case of $W=0.05$ and $D_{\mathrm{o}} / t=6.8$. As shown in Fig. 5, the cylinder with initial ovality was collapsed because initial ovality induced local collapse. Figure 6 shows the effect of initial ovality on collapse pressure. The collapse pressure decreased as the value of initial ovality increased. The trends of collapse pressure according to the initial ovality were independent of the $D_{\mathrm{o}} / t$ in the intermediate thickness range. Comparing the present FE results with the actual design pressure based on the ASME Sec. III code [17], although there is an initial ovality with $W=0.05$, the present FE collapse pressure values are much higher than the design pressure, about two times higher for the case of $D_{\mathrm{o}} / t=10$ and four times higher for the case of $D_{\mathrm{o}} / t=6.8$, so it can be concluded that the design criteria of ASME Sec. III code for the cylinder with intermediate thickness subjected to external pressure gives very conservative results.

The resulting yield locus is shown in Fig. 7 together with the present FE results for two values of $D_{\mathrm{o}} / t$. Figure 7 demonstrates that the proposed yield locus agrees well with the present $\mathrm{FE}$ results within a maximum difference of $4 \%$ in the range of interest in the present study. Thus, it can be concluded that the proposed yield locus together with the FE results can be used to predict external collapse pressure of the cylinder in an intermediate thickness range considering the interaction of plasticity and local instability due to initial ovality.

\section{APPLICATION OF PARTIAL SAFETY FACTOR}

\subsection{LRFD and PSF}

The PSF (Partial Safety Factor) concept based on the Level-1 method of the structural reliability theory was

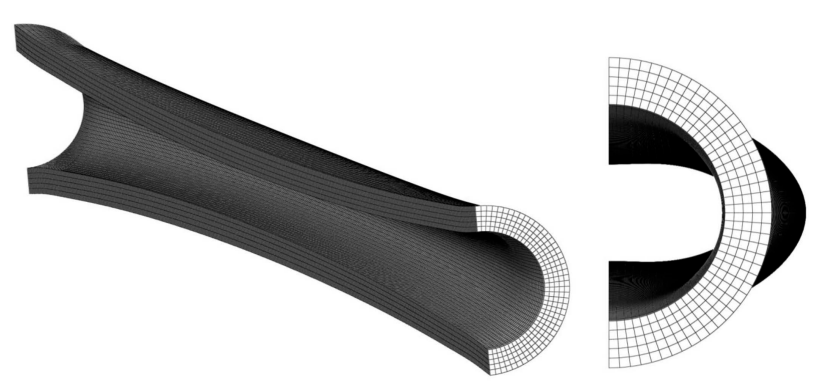

Fig. 5. Deformed Shape for the Case of $W=0.05$ and $D_{\mathrm{o}} / t=6.8$

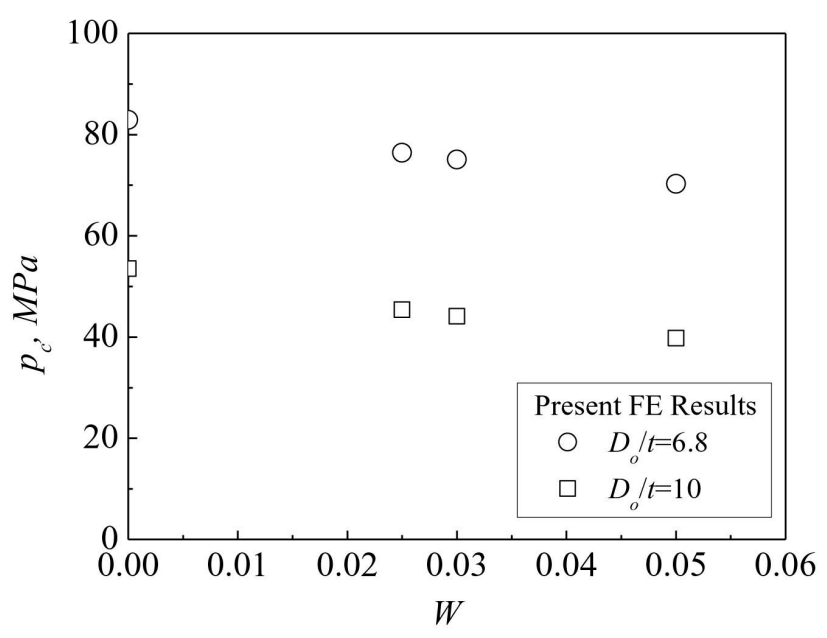

Fig. 6. Effect of Ovality on the Collapse Pressure of a Cylinder

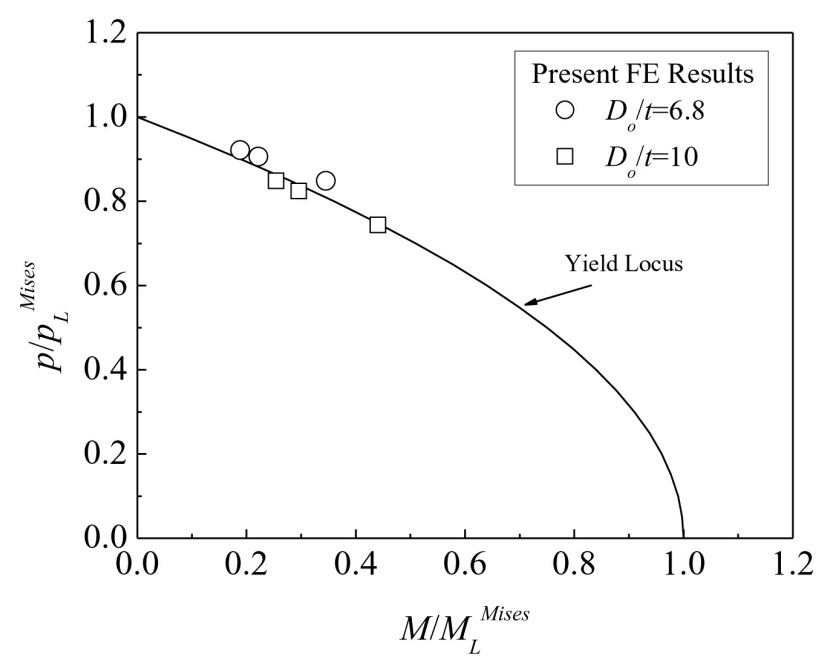

Fig. 7. Yield Locus for Cylinder with Intermediate Thickness Under Combined External Pressure and Bending 
applied for the present yield criteria locus as the examination method of the priority of importance of each parameter. LRFD (Load and Resistance Factor Design) was used to evaluate the PSF and had been used previously in civil construction engineering and petroleum industry.

Generally the judgment of a target probability of failure $\left(\mathrm{P}_{\mathrm{f}}\right)$ would be performed on the relation between the lower limit of the resistance and the upper limit of the load. The PSF concept makes it possible to allot safety factors respectively considering the uncertainty of each probabilistic variable, which constitutes a limit-state function (G) for the target probability. These basic concepts were illustrated in the L-R model of Fig. 8. The limit-state function (G) used in the LRFD can be defined as follows [18]:

$$
G=R-L
$$

$\mathrm{G}>0$ : safe, $\mathrm{G}<0$ : unsafe, $\mathrm{G}=0$ : limit-state

The resistance $(\mathrm{R})$ and the load $(\mathrm{L})$ correspond to the present yield criteria locus and a normal value inside the locus, respectively. In constituting the limit-state function using Eqs. (1) (8) in this study, the corresponding parameters are treated as probabilistic variables with a respective probabilistic distribution including a mean value and a standard deviation.
Figure 9 shows the relation between the limit-state function and the target probability of failure. The resistance -side design values $\left(\mathrm{R}_{\mathrm{i}}\right)$ and the load-side ones $\left(\mathrm{L}_{\mathrm{i}}\right)$ were evaluated to satisfy the target probability of failure indicated in Fig. 9. In order to evaluate the design point, the AFOSM method was adopted in this study. The design point is often called the MPP (Most Probably Point) and is obtained from the evaluation to satisfy the limit-load function for a target probability to the corresponding event.

According to the evaluation results of the design points, PSFs on the resistance-side and the load-side variable were defined as follows:

$$
P S F_{R}=\frac{\text { Normal Point of Re sistance }}{\text { MPP of Re sistance }}
$$

$$
P S F_{L}=\frac{\text { MPP of Load }}{\text { Normal Point of Load }}
$$

The subscript " $R$ " denotes the resistance-side and " $L$ " denotes the load-side. The normal point usually corresponds to a normal value suggested in the design specification or a mean value of probabilistic distribution.

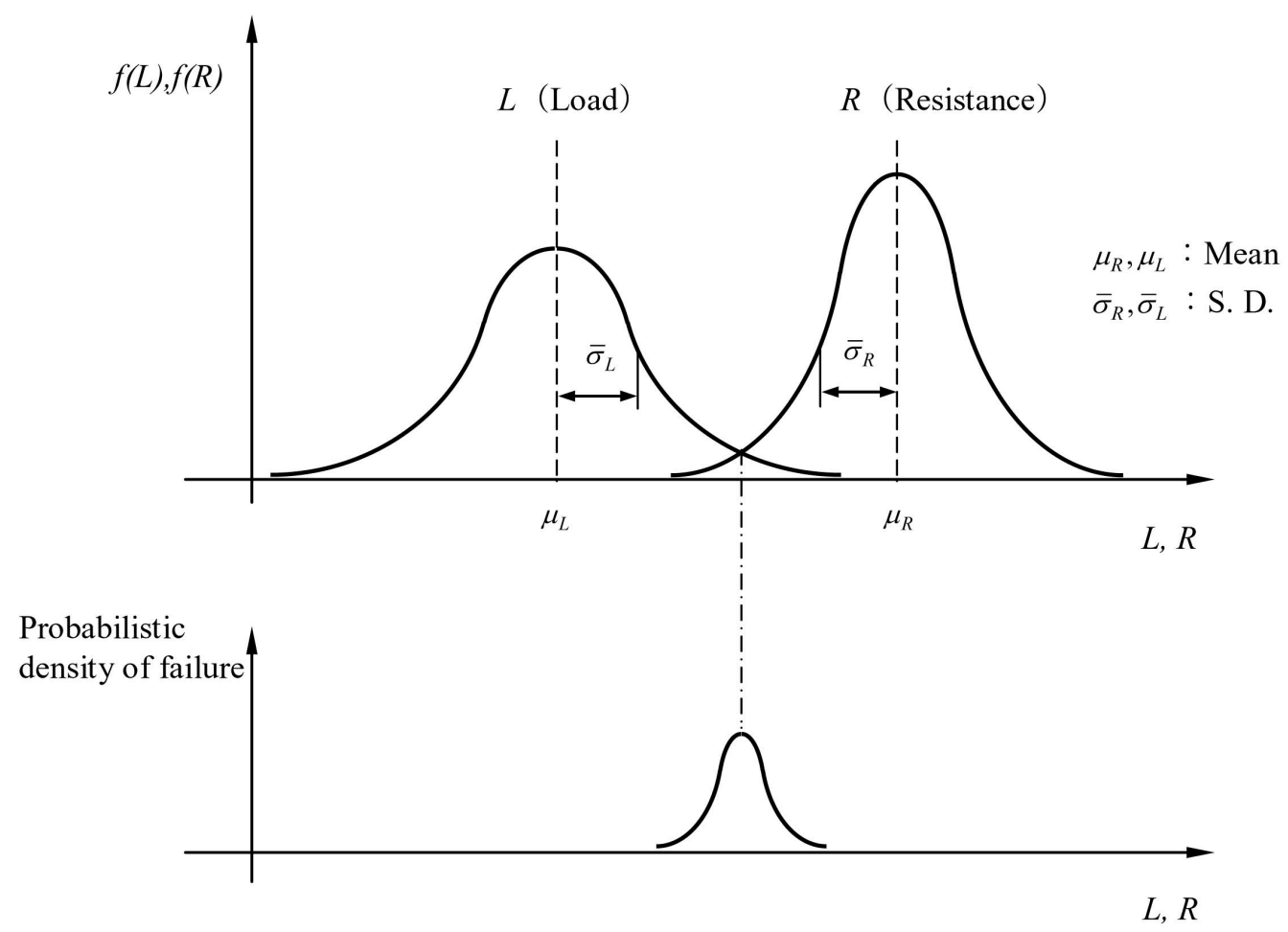

Fig. 8. L-R Model 


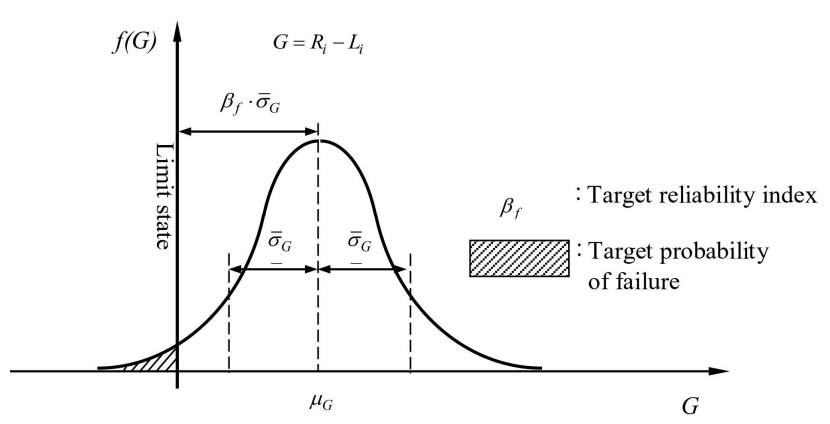

Fig. 9. Limit State Function and Target Probability of Failure

\subsection{Probabilistic Variables for Evaluation of PSF}

The above PSF concept was applied for the present collapse pressure estimation model of the SG tubes subjected to external pressure and, thus, partially evaluates the safety factors of each variable. Yield strength as a representative resistance in this study was set to the log-normal distribution referring to the research of SINTAP [19]. The Min. yield strength introduced in a material handbook [11] was adopted conservatively as the mean value of the yield strength of the SG tube (Actually the mean value is a little greater than the Min. value of the handbook. Generally, it is desirable to set the mean value of the material properties throughout the material test if not using the normal value of the design specification). SINTAP assumed a typical COV (Coefficient of Variance) of 0.08 and a COV of 0.1 for the calculation of the PSF. From the uncertainty of the material (alloy 690) of a steam generator tube in the present study, the COV was a somewhat conservatively set to 0.15 .

External pressure as a representative load was set to normal distribution referring to SINTAP. The COV of the pressure can be approximately estimated by considering the occurrence of the trip signal according to the transient event of the primary cooling system as an upper bound of reliability of $99.7 \%$. In this study, the COV of the pressure was set to 0.12 considering the trip signal of the main coolant pump. Moment was not treated as a probabilistic variable considering since it was induced by external pressure and ovality in this study. This study specifies the effect of external pressure and ovality on the SG tube failure. For this reason, the ovality was treated as an individual probabilistic variable, and its effect on the target probability of failure was investigated. The mean value of the ovality was set to 0.025 according to the ASME Code [17], and COVs of 0.1 and 0.2 were used considering the estimation error. Any other variables such as outer diameter $\left(D_{\mathrm{o}}\right)$ and thickness $(t)$ were set to the mean values of $17 \mathrm{~mm}$ and $2.5 \mathrm{~mm}$ considering the design data, and COVs of 0.01 and 0.026 were used considering the error at manufacture, respectively. The probabilistic variables treated in this study were summarized in Table 2.

\subsection{Application Results of PSF}

This study specifies the priority of importance of each parameter on the present collapse pressure estimation model by applying the PSF concept. The resistance and load factors of the limit-state function were defined as in Fig. 10 and called the "Locus method." Table 3 indicates the application results of the PSF concept when there are two types of COVs of the ovality, 0.1 and 0.2 . Generally, nuclear pressure vessel contains the occurrences of $10^{-6} /$ year for $A \& B$ events and $10^{-3} /$ year 10-2/year for C\&D events [20], so that $10^{-6}, 10^{-3}$ and $10^{-2}$ were considered to be the target probabilities of failure in this study.

From the results of PSF application, yield strength as a representative resistance can be concluded to be most important parameter to control the yielding of the corresponding SG tubes. Although the COV of yield strength was assumed to be 0.15 , which was a little conservative, the magnitude and the sensitivity of the PSFs were evaluated to be larger than any others. The external pressure was considered to be a significant parameter as a representative load. On the other hand, the sensitivity of the ovality was evaluated to be relatively not so severe, even in the COV of 0.2 , which suggests the ovality is not so effective for

Table 2. Probabilistic Variables for PSF Application

\begin{tabular}{c|c|c|c|c|c}
\hline Variables & $\begin{array}{c}\sigma_{\mathrm{y}} \\
(M P a)\end{array}$ & $\begin{array}{c}p \\
(M P a)\end{array}$ & $W$ & Normal & $\begin{array}{c}D_{0} \\
(\mathrm{~mm})\end{array}$ \\
\hline Distribution & Log-normal & Normal & Normal & 17 & 2.5 \\
\hline $\operatorname{MEAN}(\mu)$ & 212 & 17 & 0.025 & 0.01 & 0.026 \\
\hline $\operatorname{COV}$ & 0.15 & 0.12 & $0.1,0.2$ & \\
\hline
\end{tabular}




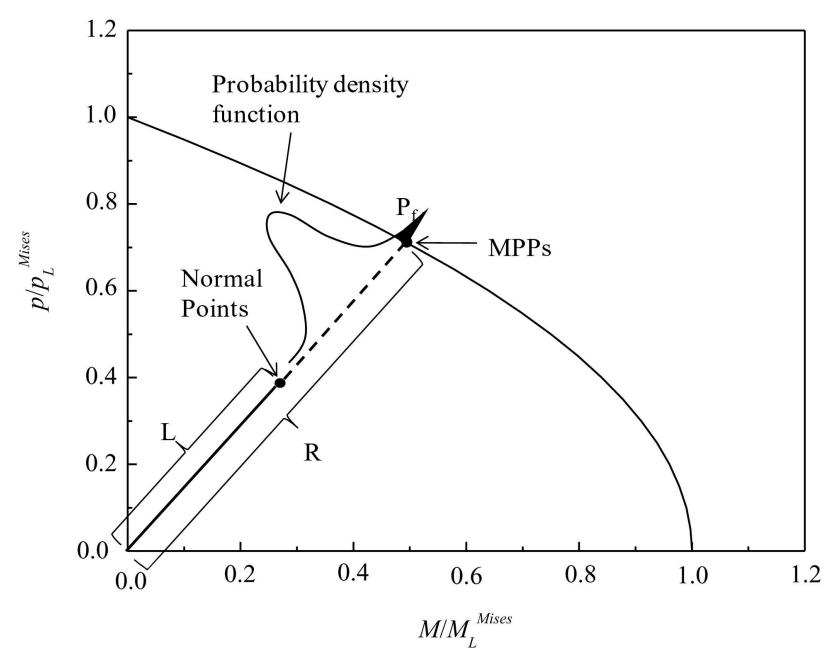

Fig. 10. Locus Method

the yield and may permit the estimation error of the ovality to some degree. The shape parameters such as the outer diameter and thickness are considered to have enough margins in the present manufacturing level, as they were not evaluated to be sensitive parameters from the results. The use of the PSFs as shown in the results of Table 3 allows the designers to guarantee the target probabilities of failure reasonably, even if not adopting the more complex probabilistic and statistical reliability theory.

\section{DISCUSSION}

In the present study, in order to propose yield locus considering the interaction of plastic collapse and local instability due to the initial ovality of the cylinder subjected to external pressure to predict collapse pressure based on a detailed FE analyses, the straight cylinder model was employed. As mentioned previously, the actual steam generator [7] consists of helically coiled tube. However, in general, the coiling radius of each tube is very large (the average value of coiling radius is about $1 \mathrm{~m}$ ), and the tubes are fully fixed by supports at regular intervals to prevent contact between tubes; the actual tube can be modeled as a straight tube. Therefore, the present results can be applied

Table 3. Results of PSF Application

. $\mathrm{COV}$ of $\mathrm{W}=0.1$

\begin{tabular}{c|c|c|c|c|c}
\hline Variables & $\sigma_{\mathrm{y}}$ & $p$ & $W$ & $D_{0}$ & $t$ \\
\hline $\mathrm{P}_{\mathrm{f}}$ & \multicolumn{5}{|c|}{ PSF } \\
\hline $\begin{array}{c}\mathrm{P}_{\mathrm{f}}=10^{-6} \\
\left(\beta_{\mathrm{f}}^{*}=4.75\right)\end{array}$ & 1.83 & 1.30 & 1.03 & 1.00 & 1.02 \\
\hline $\begin{array}{c}\mathrm{P}_{\mathrm{f}}=10^{-3} \\
\left(\beta_{\mathrm{f}}=3.09\right)\end{array}$ & 1.47 & 1.20 & 1.03 & 1.00 & 1.01 \\
\hline $\begin{array}{c}\mathrm{P}_{\mathrm{f}}=10^{-2} \\
\left(\beta_{\mathrm{f}}=2.33\right)\end{array}$ & 1.34 & 1.16 & 1.02 & 1.00 & 1.01 \\
\hline
\end{tabular}

. $\mathrm{COV}$ of $\mathrm{W}=0.2$

\begin{tabular}{c|c|c|c|c|c}
\hline Variables & $\sigma_{\mathrm{y}}$ & $p$ & $W$ & $D_{0}$ & $t$ \\
\hline $\mathrm{P}_{\mathrm{f}}$ & \multicolumn{5}{|c|}{ PSF } \\
\hline $\begin{array}{c}\mathrm{P}_{\mathrm{f}}=10^{-6} \\
\left(\beta_{\mathrm{f}}=4.75\right)\end{array}$ & 1.82 & 1.29 & 1.13 & 1.00 & 1.02 \\
\hline $\begin{array}{c}\mathrm{P}_{\mathrm{f}}=10^{-3} \\
\left(\beta_{\mathrm{f}}=3.09\right)\end{array}$ & 1.47 & 1.20 & 1.10 & 1.00 & 1.01 \\
\hline $\begin{array}{c}\mathrm{P}_{\mathrm{f}}=10^{-2} \\
\left(\beta_{\mathrm{f}}=2.33\right)\end{array}$ & 1.33 & 1.15 & 1.08 & 1.00 & 1.01 \\
\hline
\end{tabular}

$* \beta_{\mathrm{f}}$ : Target Reliability Index 
to the design of actual helically coiled tube. Another notable point is that, as described in the previous section, the yield locus of cylinder with intermediate thickness was proposed in the present study. According to Eqs. (5) (8), the $M / M_{L}^{\text {Mises }}$ due to the initial ovality of a cylinder with an intermediate thickness ranges from $\sim 0.1$ to $\sim 0.5$ (see Fig. 7). Thus, the present results can be applied to a cylinder with intermediate thickness.

In this study, the concept of partial safety factor was mainly applied to assess the priority of importance of each variable on a certain target reliability, which constitutes the limit state function for steam generator tubes subjected to external pressure. However, the present application results led to the following suggestions on the design and the evaluation viewpoint from the general intention of the PSF assessment:

- The safety factor can be organized using the evaluated PSF and the limit-state function [12, 20]. If this safety factor is lower than the resultant overall safety factor, the present design has conservation, but if not, the probabilistic model of each variable should be retreated in the sequence of the priority of importance.

- The PSFs can be exchanged with one another for a certain target reliability (for example, between a principle variable and a less variable on safety, as briefly mentioned in Section 4.3, or between a prior variable and a less variable on design price) that can offer various opportunities in the engineering component design [12].

Therefore, the present study on the yield locus and the application of the PSF concept is considered to be effective to design and perform the assessment for FFS of a cylinder subjected to external pressure.

\section{CONCLUDING REMARKS}

This paper specifies the collapse behavior of cylinders subjected to external pressure based on the detailed elastic -perfectly plastic FE analyses considering the interaction of plastic collapse and local instability due to the initial ovality of the cylinder. The cylinder with initial ovality showed the trend to collapse due to initial ovality induced local collapse. The collapse pressure decreased as the value of initial ovality increased, independently of the $D_{\mathrm{o}} / t$ in the intermediate thickness range. The proposed yield locus considering the interaction between plastic collapse and local instability for a cylinder with intermediate thickness, subjected to external pressure, agrees well with the present FE results; thus, the proposed yield locus can be utilized in the design and assessment of a cylinder under external pressure with initial ovality.

The PSF concept based on the structural reliability theory was also applied to the proposed collapse pressure estimation model, and, thus, the priority of the importance of respective parameters constituting the collapse of cylinders under external pressure was estimated in this study. From the application of the PSF concept, the yield strength was concluded to be most sensitive and the ovality was not so effective in the proposed collapse pressure estimation model.

The present results can be used for the design and performance of FFS for components composed of cylinders subjected to external pressure such as once-through type steam generators, the underwater pipelines, and so on.

\section{REFERENCES}

[1 ] Gere, J.M., 2001, "Mechanics of Materials," Fifth Edition, Brooks/ Cole.

[2 ] Wang, X., Xiao, J. and Zhang, Y.C., 2004, "A Method for Solving the Buckling Problem of a Thin-Walled Shell," International Journal of Pressure Vessels and Piping, Vol. 81, pp. 907 912.

[ 3 ] Raisuddin, K., 1998, "New Approach to Instability Testing of Shells," International Journal of Pressure Vessels and Piping, Vol. 75, No. 1, pp. 75 80.

[4] Bai, Y., Igland, R.T. and Moan, T., 1997, "Tube Collapse under Combined External Pressure, Tension and Bending," Marine Structures, Vol. 10, pp. 389 410.

[ 5 ] Tokimasa, K. and Tanaka, K., 1986, "FEM Analysis of the Collapse Strength of a Tube," ASME Journal of Pressure Vessel Technology, Vol. 108, pp. 158 164.

[6] Yeh, M.K. and Kyriakides, S., 1986, "On the Collapse of Inelastic Thick-Walled Tubes under External Pressure," ASME Journal of Energy Resources Technology, Vol. 108, pp. 35 47.

[ 7 ] Ninokata, H., 2006, “A Comparative Overview of Thermal Hydraulic Characteristics of Integrated Primary System Nuclear Reactors," Nuclear Engineering and Technology, Vol. 38, No. 1, pp. 33 44.

[8] Rahman, S., 1997, "Probabilistic Fracture Analysis of Cracked Pipes with Circumferential Flaws," International Journal of Pressure Vessels and Piping, Vol. 70, pp. 223 236.

[9] Machida, H. and Yoshimura, S., 2002, "Probabilistic Fracture Mechanics Analysis of Nuclear Piping Considering Variation of Seismic Loading," International Journal of Pressure Vessels and Piping, Vol. 79, pp. 193 202.

[10] Gupta, A. and Choi, B., 2003, "Reliability-based Load and Resistance Factor Design for Piping; An Exploratory Case Study," Nuclear Engineering and Design, Vol. 224, pp. 161 178.

[11] Avrithi, K. and Ayyub, B.M., 2010, "Load and Resistance Factor Design (LRFD) of Nuclear Straight Pipes for Loads That Cause Primary Stress," ASME Journal of Pressure Vessel Technology, Vol. 132, pp. 021101-1 021101-14.

[12] Yoo, Y.S., Huh, N.S., Kim, G.H., Jung, J.H., Kim, T.W. and Kim, J.I., 2009, "The Design and Assessment Concept of Structures Based on Partial Safety Factor,' Proceedings of the KPVP 2009 Autumn Annual Meeting.

[13] ABAQUS, Inc., User's Manual, ABAQUS Version 6.7-1, 2007.

[14] ASME Boiler \& Pressure Vessel Code Sec. II, Part D, Materials, The American Society of Mechanical Engineers, 2007.

[15] Hill, R., 1950, "The Mathematical Theory of Plasticity," Oxford University Press, pp. 248 252.

[16] Timoshenko, S.P. and Gere, J.M., 1961, “Theory of Elastic 
Stability,” McGRAW-HILL.

[17] "Rules for Construction of Nuclear Facility Components," ASME Boiler \& Pressure Vessel Code Sec. III, Division 1 Subsection NB, The American Society of Mechanical Engineers, 2007.

[18] Thoft-Christensen, P. and Baker, M.J., 1982, "Structural Reliability Theory and Its Applications," Springer-Verlag
Berlin Heidelberg New York.

[19] Partial Safety Factors for SINTAP Procedure, 2000, "OFFSHORE TECHNOLOGY REPORT," AEA Technology plc.

[20] Bloom, J.M., 2000, "Partial Safety Factors (PSF) and Their Impact on ASME Section XI, IWB-3610,” ASME Pressure Vessel and Piping Conference. 